\title{
FUNDAMENTOS DOS TESTES DE PRESSÃO EM RESERVATÓRIOS DE GÁS: DETERMINAÇÃO DO ABSOLUTE OPEN FLOW A PARTIR DO TESTE ISÓCRONO
}

\author{
O. J. ROMERO ${ }^{1}$, T. P. LIMA ${ }^{2}$ \\ Universidade Federal do Espírito Santo \\ oldrich.romero@ufes.br ${ }^{1}$ \\ Artigo submetido 25/10/2016 e aceito 17/12/2019 \\ DOI: 10.15628/holos.2019.5265
}

\section{RESUMO}

Testes de poço são realizados, normalmente, após o término da perfuração e fazem parte da avaliação exploratória de um campo de petróleo. Quando se trata de reservatórios de gás, os testes mais difundidos são os testes de contra pressão, o isócrono e o isócrono modificado. São utilizados para identificação e caracterização dos parâmetros do reservatório como o dano. Se tratando do gás é necessário levar em consideração a compressibilidade e a variação da viscosidade com a pressão e a temperatura. Este artigo tem como objetivo discutir a aplicação dos métodos analítico e mediante auxílio de um software comercial (Computer Aided Engineering - CAE) na obtenção do Absolute Open Flow - AOF a partir de um teste isócrono. A ferramenta computacional utilizada é o IHS WellTest Este tipo de teste também permite a elaboração da curva de Inflow Performance Relationship - IPR. A diferença entre os resultados obtidos a partir da aplicação dos dois métodos é de $1,7 \%$. Um melhor ajuste da linha transiente pode melhorar a precisão do resultado. A abordagem CAE torna a análise dos resultados mais rápida e fácil.

PALAVRAS-CHAVE: Teste isócrono, AOF, reservatório de gás, testes de poços, IHS WellTest.

\section{FUNDAMENTALS OF WELL TESTING IN GAS RESERVOIRS: DETERMINATION OF ABSOLUTE OPEN FLOW POTENTIAL FROM ISOCHRONAL TEST}

\begin{abstract}
Well tests are performed after completion of drilling and are part of the exploratory evaluation of an oil field. When it comes to gas reservoirs, the most widespread are the tests of back pressure tests, isochronal and modified isochronal. They are used for identification and characterization of reservoir parameters such damage. If the treating gas is necessary to take into account the compressibility and variation of viscosity with pressure and temperature. This article aims to discuss the application of theoretical and empirical methods in
\end{abstract}

getting the absolute open flow (AOF) through two approaches: analytical and computer from an isochronal test. The computational tool used was the IHS WellTest where the empirical method is implemented. This type of test also allows the construction of the IPR curve. The difference between results from the application of two methods was $1.7 \%$. The precise adjustment of transient line would improve the accuracy of the result. The computational implementation of the empirical method makes analysis faster and easier results.

KEYWORDS: Isochronal test, AOF, gas reservoir, well test, IHS WellTest. 


\section{INTRODUÇÃO}

\subsection{Testes de poços}

O teste de poço, ou também denominado teste de pressão, consiste de um conjunto de atividades que visam avaliar (qualitativamente e quantitativamente) o potencial de uma jazida petrolífera, ou seja, a sua capacidade produtiva e a valoração das suas reservas de óleo ou de gás. Testar uma formação é permitir que ela produza livremente o fluido nela contido durante um tempo determinado, medindo a pressão de fluxo de fundo de poço $\left(p_{w f}\right)$ em frente aos canhoneados (interface reservatório/poço produtor) e a vazão de fluidos $(q)$ normalmente em superfície, para diversos instantes de tempo. Os tempos de aquisição dos dados dependem da finalidade do teste, de forma geral são denominados taxa de amostragem que pode variar de alguns segundos a vários minutos. O processamento e a interpretação deste conjunto de dados (vazão, pressão e tempo) é o fundamento do processo de testes de poço. Uma representação esquemática do exposto pode ser visualizada na Figura 1, na qual se destacam dois dos principais testes desenvolvidos na indústria de petróleo para vazão constante, o drawdown, que corresponde a um período de fluxo, e o buildup, que corresponde a um período de estática.
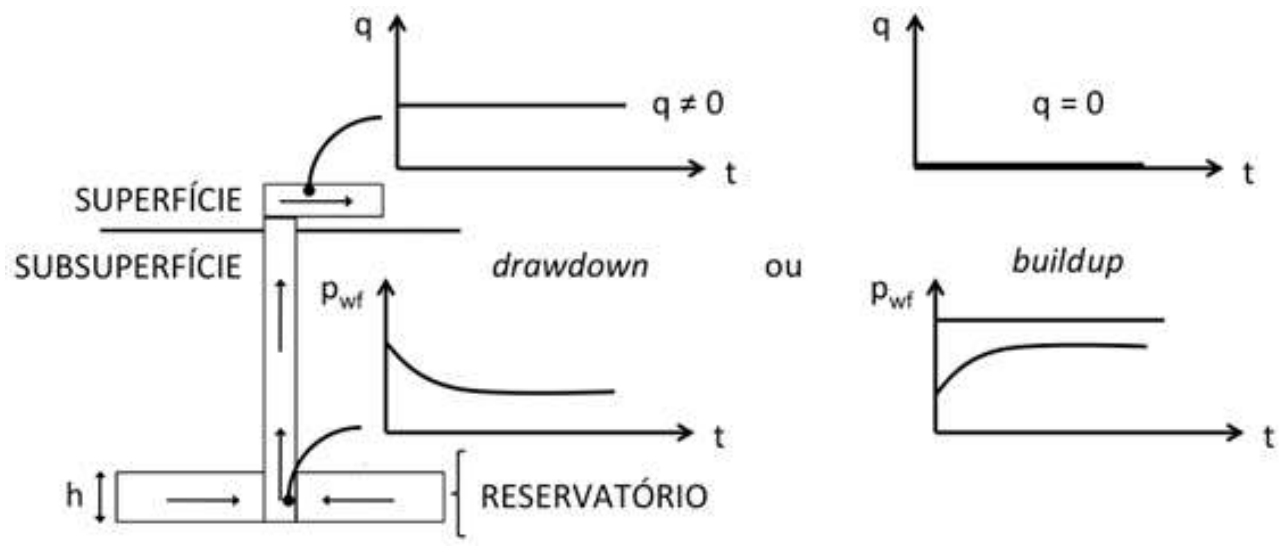

Figura 1: Representação gráfica do testes de poço: a pressão $p_{w f}$ é medida em frente aos canhoneados e a vazão $q$ em superfície para diversos instantes de tempo $t$ (Fonte: Romero \& Garuzzi, 2015).

O drawdown consiste na medição da pressão de fundo de poço em um determinado intervalo de tempo e produzindo a uma vazão constante. Normalmente, o poço está em um período de shut in (ou estática) antes do teste em fluxo, durante um período de tempo suficiente para permitir que a pressão equalize ao longo da formação, para atingir a pressão estática. 0 buildup é realizado para uma vazão constante e nula, com isso a pressão de fundo de poço cresce com o tempo até atingir o equilíbrio, ou seja, um valor único para todo o reservatório $O$ principal objetivo é determinar a pressão estática do reservatório (Ahmed \& Meehan, 2012; Garuzzi \& Romero, 2014).

Os testes de pressão são realizados com diversas finalidades. Estes objetivos são definidos de acordo com o estágio de vida do reservatório de petróleo ou de gás. Por exemplo, se foi recém descoberto ou já está em produção. De uma forma geral, cita-se como principais objetivos:

- identificar os fluidos presentes na formação; 
- avaliar a capacidade produtiva da formação (taxas econômicas de produção);

- determinar a permeabilidade absoluta da rocha porosa;

- determinar o fator de película ao redor do poço;

- identificar as fronteiras do reservatório assim como tamanho e forma;

- determinar a pressão inicial ou a pressão média volumétrica do reservatório;

- descobrir a existência ou não de descontinuidades (falhas, barreiras de permeabilidade, etc.);

- determinar a extensão de fraturas e outras características geométricas do reservatório, dente outros.

Os testes podem ser realizados em todas as fases da vida produtiva de um reservatório com objetivos distintos. Em um poço exploratório ou pioneiro (exploration well) os testes irão confirmar a existência de hidrocarbonetos, natureza do fluido, índice de produtividade e as propriedades do reservatório. Em um poço de delineamento ou de extensão (appraisal well) os testes são utilizados para refinar dados prévios, realizar testes mais longos, amostragem dos fluidos, pressão inicial do reservatório. Já em um poço de desenvolvimento (development well) os testes são realizados para verificar a necessidade de estimulação dos poços, pressão média volumétrica. Ou seja, os testes são realizados em todas as etapas da vida útil de um reservatório (Romero, 2015).

Os testes de poço se iniciam com a obtenção dos dados de pressão $p_{w f}$ e vazão $q$ normalmente realizados por empresas especializadas. Estes dados são analisados com o auxílio da solução da equação da difusividade, seja para fluidos moderadamente compressíveis ou compressíveis. Esta expressão é o fundamento para interpretar as características do reservatório de espessura $h$ e calcular seus parâmetros. Diversas formas de apresentar os resultados do teste são oferecidas na literatura. Destaca-se dentre eles o procedimento de Horner para o buildup, no qual o tempo do teste $\Delta t$ é trabalhado e reapresentado como $\log \left(\frac{t_{p}+\Delta t}{\Delta t}\right)$, onde $t_{p}$ é o tempo de produção em que o poço permanece aberto (drawdown) antes do fechamento. Horner propôs o gráfico semi-logarítmico $p_{w s}$ vs. $\log \left(\frac{t_{p}+\Delta t}{\Delta t}\right)$, onde sua inclinação $m$ permite determinar a permeabilidade $k$ mediante $k=162,6 \frac{q B \mu}{m h}$ e, posteriormente com a solução da equação da difusividade, o fator de película $s$. $B$ é o fator volume de formação do óleo de viscosidade $\mu$.

De forma simplificada, o processo do teste envolve quatro etapas, sendo elas: a) planejamento do teste, b) execução do teste, c) interpretação dos dados coletados e d) apresentação de resultados, cada uma com seus próprios desafios e dificuldades (Romero \& Garuzzi, 2015). Essa sequência é apresentada esquematicamente na Figura 2, e descrita brevemente nos próximos parágrafos.

- Planejamento do teste: para cada informação que se pretende extrair do reservatório, um tipo específico de teste deve ser realizado. Desse modo o planejamento se faz uma etapa crítica de todo o processo;

- Execução do teste: registro da pressão no fundo do poço e da vazão na superfície para diversos instantes de tempo.

- Interpretação dos dados coletados: processamento dos dados utilizando gráficos semi-logarítmicos, gráficos logarítmicos, método de Horner, método MDH (Miller, Dyes, \& Hutchinson, 1950), método MBH (Matthews, Brons, \& Hazebroek, 1954), 
derivada de Bourdet (Bourdet, Whittle, Douglas, \& Pirard, 1983), curvas tipo (Agarwal, Al-Hussainy, \& Ramey, 1970; Gringarten et al., 1979), interpretação automatizada via softwares, etc.;

- Resultados: índice de produtividade, pressão inicial do reservatório, pressão média do reservatório, depleção, permeabilidade, fator de película, fraturas, geometria do reservatório, coeficiente de estocagem, regime do escoamento, raio de drenagem, identificação de fluidos, etc.

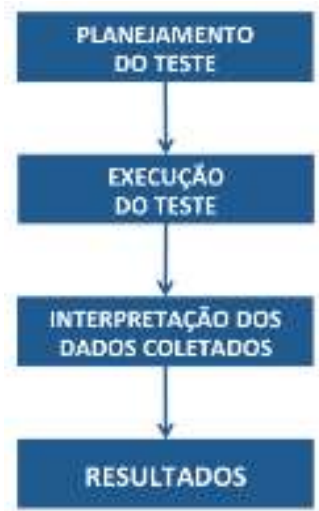

Figura 2: Processo simplificado do teste de poço (Romero e Garuzzi, 2015).

O registro de dados na etapa de execução do teste é fortemente dependente de experiência de campo do Engenheiro, assim como sua habilidade para lidar com equipamentos de forma organizada e responsável. Os conceitos teóricos do escoamento em meios porosos começa a ser cada vez mais importante quando da interpretação dos dados coletados. Na Figura 3 esta sequência de eventos é destacada em que a solução da equação da difusividade (modelo matemático) é parte fundamental no processo.

As etapas principais do teste

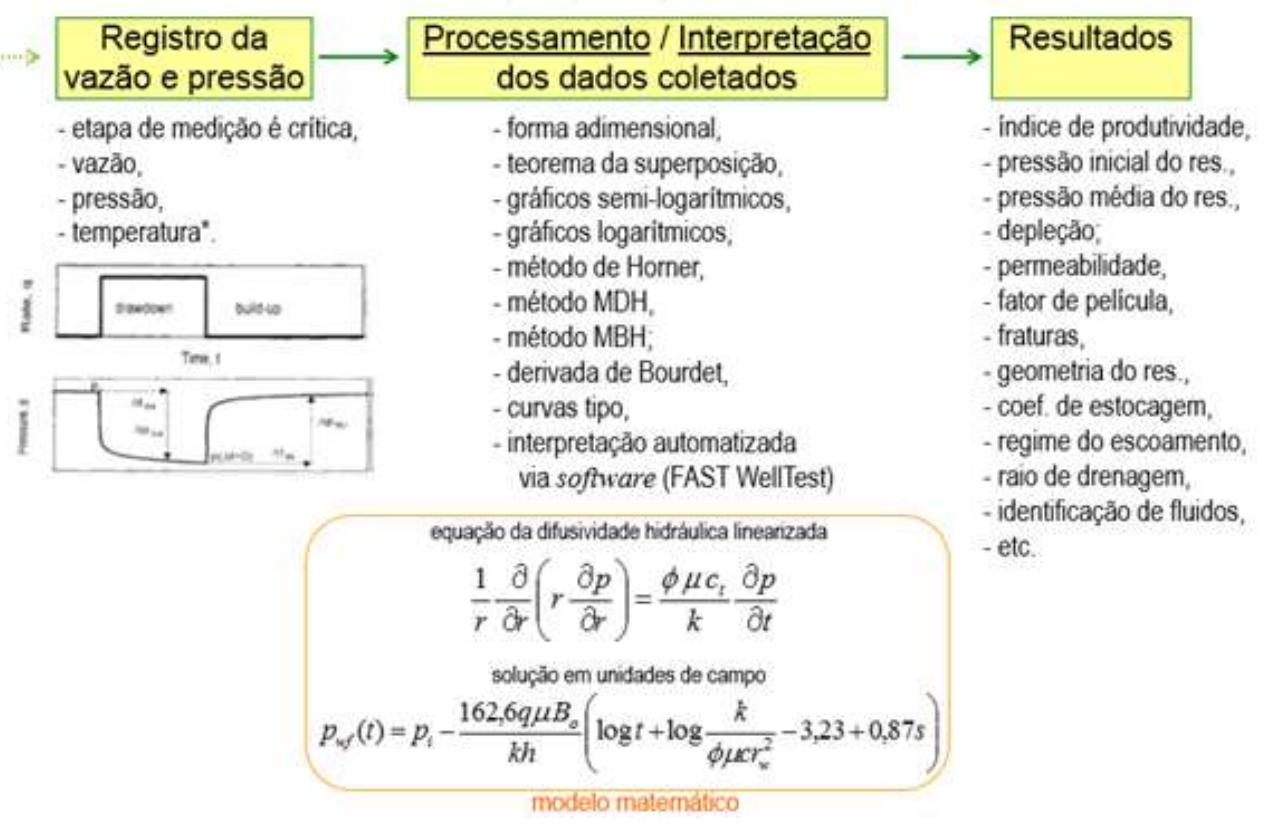

Figura 3: As etapas principais do teste de poço (Romero \& Garuzzi, 2015). 


\subsection{Objetivo}

Apresentar a fundamentação teórica dos testes de poço para reservatórios de gás e mostrar sua aplicação na avaliação do AOF (Absolute Open Flow) mediante o teste isócrono. A análise é realizada a partir de uma comparação de resultados obtidos pelas abordagens analítica e computacional.

\section{ESCOAMENTO DE GASES EM MEIOS POROSOS}

\subsection{Soluções da equação da difusividade hidráulica}

A equação para o estudo do comportamento do fluxo de fluidos em meios porosos é a equação da difusividade hidráulica. A partir dela são desenvolvidas soluções para as diversas situações em que o reservatório pode se encontrar, sendo possível aplicações em testes de poços. A equação da difusividade é derivada da lei da conservação de massa, da lei de Darcy e equações de estado. Hipóteses simplificadoras devem ser atendidas: reservatório homogêneo e isotrópico de espessura constante; um único poço produtor de raio $r$ muito pequeno $r \rightarrow 0$ localizado no centro do reservatório completado ao longo de toda a espessura (Figura 4); reservatório muito grande ("infinito") implicando condições transientes; fluido monofásico; efeitos da gravidade desprezíveis; permeabilidade constante. O fluxo é transiente quando a perturbação na pressão causada pela produção ainda não atinge a fronteira do reservatório ou quando as interferências de outros poços não tem produzidos efeitos significativos.

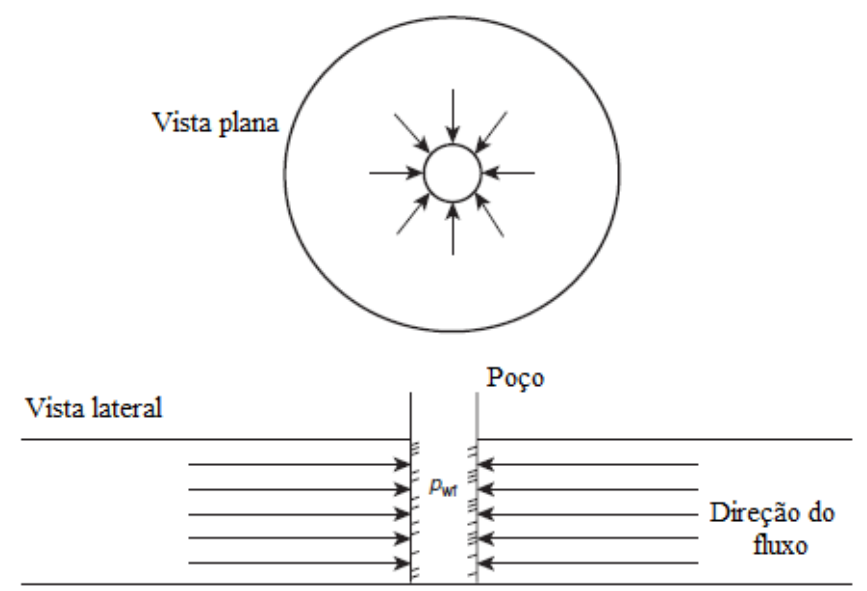

Figura 4: Fluxo radial em um reservatório (Ahmed \& Meehan, 2012).

Com estas considerações é obtida a equação geral que descreve o fluxo de fluidos em uma direção radial $r$ como sendo $\frac{1}{r} \frac{\partial}{\partial r}\left(\frac{k}{\mu} \rho r \frac{\partial p}{\partial r}\right)=\rho \phi c_{t} \frac{\partial p}{\partial t}+\rho \frac{\partial \rho}{\partial t}$. Quando se trata de um fluido compressível, como é o caso do gás, alguns parâmetros são afetados pelas variações de pressões durante seu percurso. Sendo assim a formulação para a massa específica e a compressibilidade do gás são $\rho=\frac{p M}{Z R T}$ e $c_{g}=\frac{1}{p}-\frac{1}{Z} \frac{d Z}{d p}$, respectivamente. Onde $p$ e $T$ são, respectivamente, a pressão e a temperatura em qualquer ponto do reservatório, $M$ é a massa molar do gás, $Z$ é o fator de compressibilidade e $R$ é a constante universal dos gases. Combinando estas relações tem-se a equação da difusividade para fluidos compressíveis:

$\frac{1}{r} \frac{\partial}{\partial r}\left(r \frac{p \mu}{Z} \frac{\partial p}{\partial r}\right)=\frac{\emptyset \mu c_{t}}{k} \frac{p}{\mu Z} \frac{\partial p}{\partial t}$ 
onde $k$ é a permeabilidade, $\mu$ é a viscosidade, $\phi$ é a porosidade, $c_{t}$ é a compressibilidade total (soma das compressibilidades dos fluidos presentes no reservatório e da formação), depende da saturação de cada fluido).

Existem três soluções para a equação apresentada, uma exata e duas aproximadas, todas utilizam o conceito de pseudopressão do gás real $m(p)$, desenvolvido por Al-Hussainy e Ramey (1966), cuja expressão é

$m(p)=2 \int_{p_{0}}^{p} \frac{p}{\mu Z} d p$,

em que $p_{0}$ é uma pressão de referência. A solução exata obtida por Al-Hussainy e Ramey (1966), denominada método $m(p)$, é

$m\left(p_{w f}\right)=m\left(p_{r}\right)-57.895,3\left(\frac{p_{s c}}{T_{s c}}\right)\left(\frac{q_{g} T}{k h}\right)\left[\log \left(\frac{k t}{\emptyset \mu_{g} c_{t} r_{w}^{2}}\right)-3,23+0,87 s\right]$,

$p_{w f}$ é a pressão de fluxo no fundo do poço (psi), $p_{r}$ é a pressão do reservatório (psi), $q_{g}$ é a vazão de gás (Mscf/d), $t$ é o tempo em horas, $k$ é a permeabilidade $(\mathrm{mD}), P_{s c}$ é a pressão a condições standard (psi), $T_{s c}$ é a temperatura a condições standard $\left({ }^{\circ} \mathrm{R}\right), r_{w}$ é o raio do poço (ft), $h$ é a espessura do reservatório (ft), $\mu_{g}$ é a viscosidade do gás a pressão do reservatório $(\mathrm{cP}), c_{t}$ é o coeficiente de compressibilidade total a $p_{r}\left(\mathrm{psi}^{-1}\right), \phi$ é a porosidade, $s$ é o fator de película e $T$ é a temperatura do reservatório $\left({ }^{\circ} \mathrm{R}\right)$. $\mathrm{O}$ fator de película é um modelo matemático que incorpora situações físicas como dano ou estimulo, quantificando a diferença entre a queda de pressão prevista pela lei de Darcy e a queda de pressão real. Para um poço danificado uma restrição ao fluxo está presente ao redor do poço, isto produz uma queda de pressão adicional para o fluxo no sentido reservatório/poço. Já a estimulação melhora o escoamento para o poço.

Esta solução não é simples de ser utilizada uma vez que tabelas são necessárias para recuperar a pressão $p$ a partir da psudopressão $m(p)$. Duas aproximações para superar esta dificuldade são utilizadas, com a escolha dependente da pressão. Al-Hussainy e Ramey (1966) mostraram que quando a pressão é inferior a 2.000 psia, o produto $\mu Z$ é quase constante (Figura $5 a)$ e pode ser retirado da integral da equação original, Equação (2), resultando em $m(p)=$ $\frac{2}{\bar{\mu} \bar{Z}} \int_{p_{0}}^{p} p d p$, tal que $\bar{\mu}$ e $\bar{Z}$ são a viscosidade e o fator de compressibilidade do gás avaliados a uma pressão média, $\bar{p}$, dada por $\bar{p}=\sqrt{\frac{p_{r}^{2}+p_{w f}^{2}}{2}}$

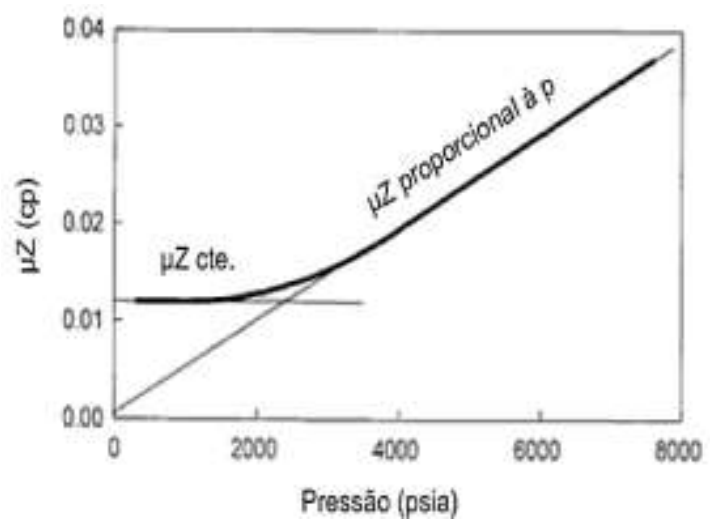

a)

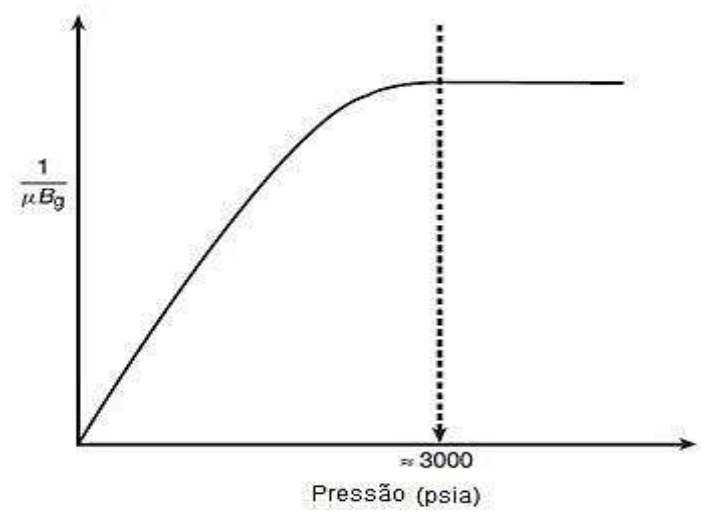

b)

Figura 5: a) Variação de $\mu Z$ com a pressão (Bourdet, 2002); b) Variação de $1 /\left(\mu B_{g}\right)$ com a pressão (Ahmed \& Meehan, 2012). 
Esta aproximação, denominada método $p^{2}$, permite obter a solução diretamente em função da pressão $p_{w f}$ e não mais dependendo da pseudo pressão $m(p)$ :

$p_{w f}^{2}=p_{r}^{2}-\left(\frac{1.637 q_{g} T \bar{\mu} \bar{z}}{k h}\right)\left[\log \left(\frac{k t}{\emptyset \mu c_{t} r_{w}^{2}}\right)-3,23+0,87 s\right]$.

A segunda aproximação, sugerida por Fetkovich (1973), é para pressões acima de 3.000 psia, quando o termo $1 /\left(\mu B_{g}\right)$ é aproximadamente constante (Figura $\left.5 \mathrm{~b}\right)$ :

$p_{w f}(t)=p_{r}-\left(\frac{162,5 \times 10^{-3} q_{g} \bar{\mu} \bar{B}_{g}}{k h}\right)\left[\log \left(\frac{k t}{\emptyset \mu c_{t} r_{w}^{2}}\right)-3,23+0,87 s\right]$,

as propriedades médias do gás, $\bar{\mu}$ e $\bar{B}_{g}$, são avaliadas na pressão média $\bar{p}$ definida por $\bar{p}=\frac{p_{r}+p_{w f}}{2}$.

Quando a perturbação atinge a fronteira o transiente termina e se origina o regime pseudopermanente. Esta condição ocorre quando o declínio temporal de pressão em qualquer ponto se mantém constante, isto é $\left(\frac{\partial p}{\partial t}\right)_{i}=$ constante. Partindo de $q t=V_{p}\left[p_{i}-\bar{p}(t)\right] c_{t}$ (onde $q$ é a vazão, $t$ o tempo, $v_{p}$ é o volume poroso, $p_{i}$ é a pressão inicial do reservatório e $\bar{p}$ é a pressão média do reservatório) Rosa, Carvalho e Xavier (2006) demonstram a equação para este regime

$\frac{1}{r} \frac{\partial}{\partial r}\left(r \frac{\partial m(p)}{\partial r}\right)=-\frac{2 q_{g} T p_{s c}}{\pi r_{e}^{2} h \emptyset c_{t} T_{s c}}$

ainda como não há fluxo na fronteira externa, $\partial m(p) / \partial r=0$ em $r=r_{e}$, a equação é resolvida para obter o diferencial de pseudopressão $m\left(p_{r}\right)-m\left(p_{w f}\right)=\frac{q_{g} T p_{s c}}{\pi k T_{s c}}\left(\ln \frac{r_{e}}{r_{w}}-\frac{1}{2}\right)$.

Por outro lado, de $m(p)=\frac{2}{r_{e}^{2}-r_{w}^{2}} \int_{r_{w}}^{r_{e}} m\left(p_{r}\right) d r$ obtém-se a solução exata para a equação da difusividade no regime pseudopermanente em termos da pressão média do reservatório $m\left(\bar{p}_{r}\right)-m\left(p_{w f}\right)=\frac{q_{g} T p_{s c}}{\pi k T_{s c}}\left(\ln \frac{r_{e}}{r_{w}}-\frac{3}{4}+s\right)$.

Utilizando os conceitos apresentados, quando a pressão do reservatório é menor que 2.000 psia e fazendo $p_{s c}=14,7$ psia e $T_{s c}=520^{\circ} \mathrm{R}$ tem-se a solução aproximada pelo método $p^{2}$ para o escoamento de gases em regime pseudopermanente

$p_{w f}^{2}=\bar{p}_{r}^{2}-\frac{1.422 q_{g} \bar{\mu}_{g} \bar{z}}{k h}\left[\ln \left(\frac{r_{e}}{r_{w}}\right)-\frac{3}{4}+s\right]$.

Similarmente, utilizando o método $p$ no regime pseudopermanente, obtêm-se

$p_{w f}=\bar{p}_{r}-\frac{7,08 \times 10^{-6} q_{g} \bar{\mu}_{g} \bar{B}_{g}}{k h}\left[\ln \left(\frac{r_{e}}{r_{w}}\right)-\frac{3}{4}+s\right]$.

O fluxo turbulento é causado pelo incremento da velocidade do gás à medida que este se aproxima do poço com o fluido apresentando uma queda de pressão adicional significativa. Em termos da pseudopressão, e de acordo com Wattenburger e Ramey (1968), é representado como $\Delta m(p)_{n a ̃ o-D a r c y}=3,161 \times 10^{-12}\left[\frac{\beta T \gamma_{g}}{\mu_{g w} h^{2} r_{w}}\right] q_{g}^{2}$, onde $q_{g}$ é a vazão de gás $(M s c f / d)$, $\mu_{g w}$ é a viscosidade $(c P)$ do gás avaliada na $p_{w f}, \gamma_{g}$ é a densidade do gás, $h$ é a espessura do reservatório (ft) e $\beta$ é o coeficiente turbulento de velocidade $\left(\mathrm{ft}^{-1}\right)$ determinado por $\beta=\frac{2,33 \times 10^{-10}}{k^{1,2}}$. Utilizando o coeficiente de fluxo turbulento $D$ dado por $D=\frac{F k h}{1422 T}$, e $F$ o coeficiente de fluxo não-darciano igual a $F=3,161 \times 10^{-12}\left[\frac{\beta T \gamma_{g}}{\mu_{g w} h^{2} r_{w}}\right]$, tem-se $\Delta m(p)_{n a ̃ o-D a r c y}=F q_{g}^{2}$. Este coeficiente é 
adicionado ás soluções da equação da difusividade hidráulica, conforme destacado na próxima subseção.

\subsection{Teste isócrono}

É um teste aplicado somente para reservatórios de gás. Reservatório de gás é a jazida que contém uma mistura de hidrocarbonetos que se encontram no estado gasoso nas condições de reservatório. Podem ser classificados como reservatórios de gás seco, reservatórios de gás úmido e reservatórios de gás retrógrado, dependendo do comportamento do fluido quando sujeito a reduções de pressão dentro do próprio reservatório e também do tipo de fluido resultante nos equipamentos de superfície (Rosa et al., 2006).

O teste isócrono pode ser dividido em duas etapas. Na primeira têm-se diversos fluxos em intervalos de tempo constante e o raio de investigação $\left(r_{i}\right)$ é menor que o raio do reservatório $\left(r_{e}\right)$, predominando o regime transiente. Na segunda etapa tem-se o fluxo estendido, onde o raio de investigação é maior ou igual ao raio do reservatório, ou seja, a partir desse momento tem-se a influencia das condições de fronteira, estabelecendo-se o regime pseudopermanente.

Este teste é do tipo multi-vazão, significando que é formado por diversos períodos de fluxo intercalados com períodos de estática (shut in) ou de vazão nula. Os períodos de fluxo são iguais. Já os períodos de estática são mantidos por um tempo suficiente para que a pressão medida no fundo do poço alcance condições essencialmente estáticas. Além disso, uma etapa final de fluxo estendido, tempo suficiente para alcançar a estabilização da pressão, é necessária. Como o teste se aplica para reservatórios com permeabilidade baixa, o raio de investigação $r_{i}$ normalmente não atinge a fronteira do reservatório $r_{e}$, portanto $r_{i}<r_{e}$, implicando condição transiente. O teste é importante pois é executado sem a necessidade de que a produção seja interrompida por tempos muito longos, tal que não seja necessário atingir as condições de estabilidade em cada vazão. Como os períodos de fluxo são iguais, o raio de investigação será o mesmo ao fim de cada etapa (Figura 6).

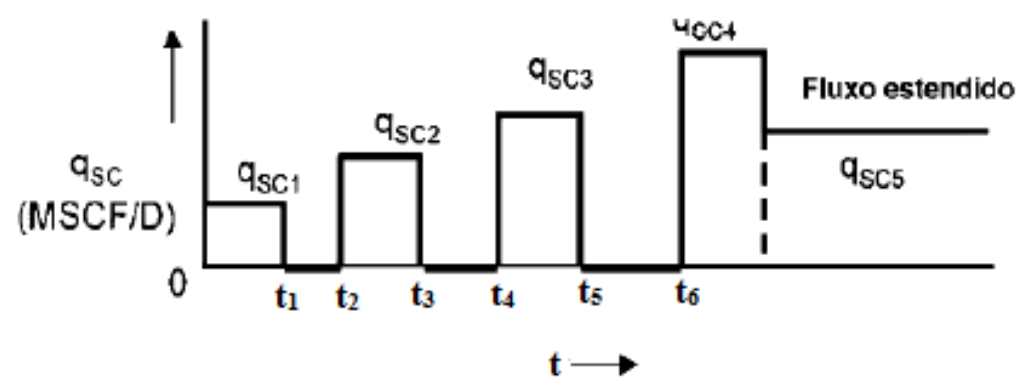

(a)

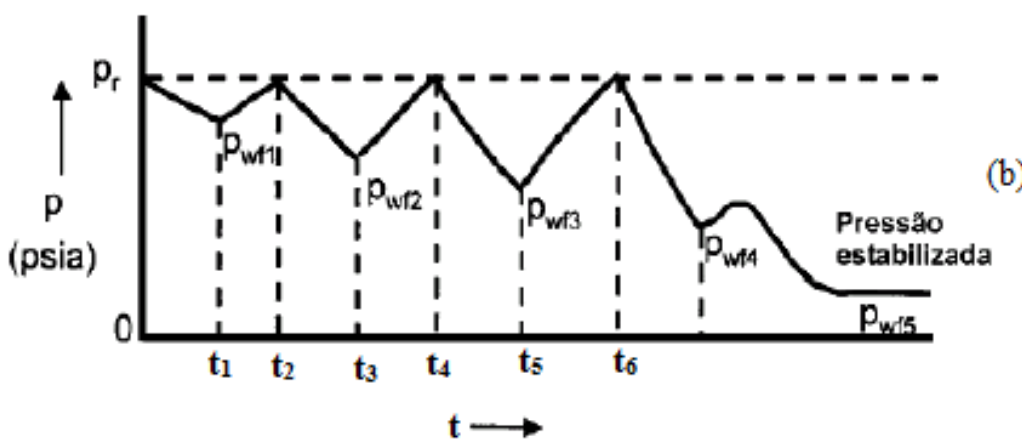

Figura 6: Teste isócrono: (a) vazão versus tempo, (b) pressão versus tempo (modificado de Guiteras, 2003). 
Na Figura 6 são apresentados os períodos de fluxo $\left(q_{s c}\right)$ para diversos instantes de tempo e a resposta da pressão $(p)$, onde:

- Os períodos de fluxo, exceto o último, são iguais $\left(t_{1}=t_{3}-t_{2}=t_{5}-t_{4}\right)$;

- Os períodos de estática tem o objetivo de deixar $p \cong \bar{p}\left(t_{2}-t_{1} \neq t_{4}-t_{3} \neq t_{6}-t_{5}\right)$;

- Um período de fluxo final no qual o poço estabiliza-se é desejável, mas não essencial.

Este teste é usado para estimar o Absolute Open Flow (AOF), que é a vazão teórica a qual o poço produziria caso a pressão de fundo de poço fosse zero. O AOF é usado como uma medida do desempenho da produção do gás, pois quantifica a habilidade do gás se movimentar em direção ao poço. O teste permite também construir a curva de IPR (Inflow Performance Relationship) que relaciona a pressão no fundo do poço com a vazão de fluidos produzidos em condições de superfície.

A teoria mais geral do teste isócrono é apresentada normalmente em termos da pseudopressão $m(p)$, entretanto no presente artigo a teoria será abordada em termos da aproximação para baixas pressões denominada método $p^{2}$, isso porque o interesse é efetuar a comparação com a resposta obtida via simulador numérico comercial. Neste contexto, para os regimes transiente e pesudopermanente as Equações (4) e (7) são reescritas incluindo o efeito da turbulência, parâmetro $D$, como sendo:

a) Solução para regime transiente $\left(r_{i}<r_{e}\right)$

$p_{w f}^{2}=\bar{p}^{2}+1.637 \frac{\bar{\mu} \bar{Z} T q_{g}}{k h}\left[\log \left(\frac{1.688 \phi \bar{\mu} c_{t} r_{w}^{2}}{k t}\right)-\left(\frac{s+D q_{g}}{1,151}\right)\right]$,

que é reescrita de forma compacta como

$\bar{p}^{2}-p_{w f}^{2}=a_{t} q_{g}+b q_{g}^{2} \quad$ ou $\quad \frac{\bar{p}^{2}-p_{w f}^{2}}{q_{g}}=a_{t}+b q_{g}$

onde $a_{t}=1.422 \frac{\bar{\mu} \bar{Z} T}{k h}\left[\frac{1}{2} \ln \left(\frac{k t}{1.688 \phi \bar{\mu} c_{t} r_{w}^{2}}\right)+s\right]$.

b) Solução para regime estabilizado $\left(r_{i} \geq r_{e}\right)$

$p_{w f}^{2}=\bar{p}^{2}-1.422 \frac{\bar{\mu} \bar{z} T q_{g}}{k h}\left[\ln \left(\frac{r_{e}}{r_{w}}\right)-\frac{3}{4}+\left(s+D q_{g}\right)\right]$,

escrita de forma compacta tem-se

$\bar{p}^{2}-p_{w f}^{2}=a q_{g}+b q_{g}^{2}, \quad$ ou $\quad \frac{\bar{p}^{2}-p_{w f}^{2}}{q_{g}}=a+b q_{g}$

os termos $a$ e $b$ são determinados segundo o procedimento detalhado na subseção §4.1.

Em reservatórios com permeabilidade muito baixa o fluxo estabilizado não é alcançado em um período de tempo razoável. Assim, a curva IPR é obtida a partir de equações teóricas para o fluxo transiente. Segundo Lee (1984), obtém-se resultados razoavelmente satisfatórios utilizando um gráfico de $\log \left(\bar{p}^{2}-p_{w f}^{2}\right)$ vs. $\log q_{g}$ que resulta na equação de uma reta dada por

$q_{g}=C\left(\bar{p}^{2}-p_{w f}^{2}\right)^{n}$,

onde $C$ e $n$ representam as propriedades do sistema rocha-fluido e geometria do reservatório, relacionados com a inclinação da reta. Neste artigo é abordado o teste onde a última vazão é mantida até atingir o ponto de estabilização. 


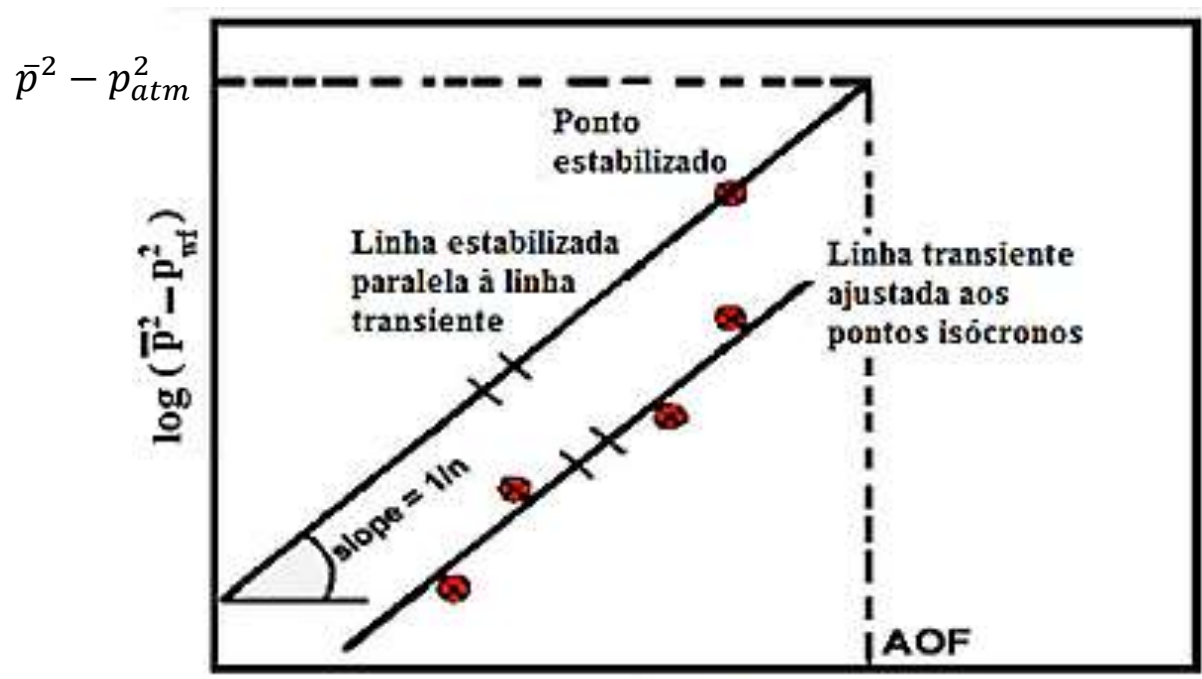

$\log \mathbf{q g}$

Figura 7: Esquema para teste de fluxo isócrono (Modificado de Guiteras, 2003).

Os períodos de fluxo e de estática fornecem as informações necessárias para traçar quatro pontos, como pode ser visto na Figura 7. Uma linha reta, chamada de linha de entregabilidade transiente é traçada através destes quatro pontos. A duração da última vazão é prorrogada até a resposta da pressão se estabilizar, ou seja, até que a pressão $\bar{p}$ seja atingida. Esta informação é usada para traçar uma linha paralela à linha de entregabilidade transiente através do ponto estabilizado, que é chamada de linha de entregabilidade estabilizada. 0 expoente $n$ é determinado a partir da inclinação da curva. O parâmetro $C$ é determinado usando $n$ e as coordenadas de pressão e vazão de qualquer ponto estabilizado na linha de entregabilidade.

Por outro lado, para estimar o absolute open flow (AOF) é traçada uma linha paralela entre o ponto estabilizado (pseudopermanente), ou seja, quando $\left(r_{i} \geq r_{e}\right)$, e os pontos de tempos iguais transientes. A partir do ponto $\bar{p}^{2}-p_{a t m}^{2}$ no eixo vertical, é traçada uma reta paralela ao eixo horizontal até a linha que contém o ponto estabilizado, a vazão correspondente a esse ponto de encontro é o AOF, conforme apresentado na Figura 7. Com $C$ e $n$ e a Equação (14), temse a curva IPR - Inflow Performance Relationship representada na Figura 8. Esta curva é utilizada para prever o comportamento do fluxo do reservatório para o poço.

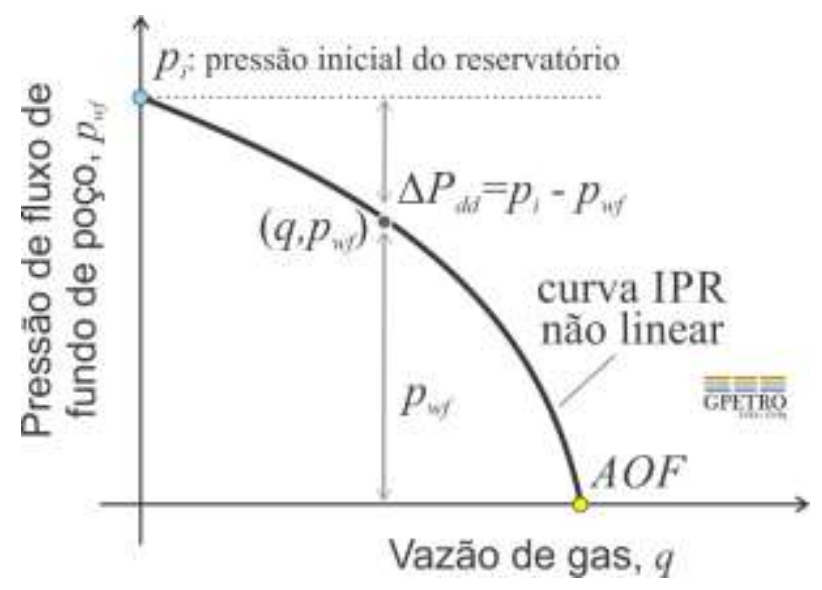

Figura 8: Representação gráfica da curva IPR. 
O estudo sobre testes de poço para reservatórios de gás está crescendo em decorrência da explotação do gás de xisto e da crescente demanda de energia. Outros testes, como o isócrono modificado, são citados em Lee (1984), Bourdet (2002), Romero e Garuzzi (2015) e HIS (2015).

\section{METODOLOGIA DE SOLUÇÃO}

A teoria apresentada é aplicada para analisar o AOF de um reservatório portador de gás a partir dos dados de um teste isócrono. Os dados, mostrados na Tabela 1 e adaptados dos apresentados por Lee (1984), são formados pela leitura de pressões e vazões para diversos instantes de tempo. O poço se encontra inicialmente fechado (shut in inicial) durante $48 \mathrm{~h}$ mantendo uma pressão de 13,459 kPa, posteriormente é colocado para produzir a uma vazão constante de 73,482 m³/d (2,6 MMSCF/D; com MMSCF/D = Million Standard Cubic Feet per Day) por $12 \mathrm{~h}$ sendo a pressão (de fluxo, $p_{w f}$ ) a nível dos canhoneados de 12,142 kPa. Esta etapa é o primeiro fluxo (drawdown), o qual é seguido de um primeiro fechamento (shut in) de $15 \mathrm{~h}$ em que a pressão (estática, $p_{w s}$ ) medida no fundo do poço se recupera alcançando $13,459 \mathrm{kPa}$. $\mathrm{O}$ processo é repetido por mais dois períodos de fluxo e de estática, seguido de um fluxo estendido de $72 \mathrm{~h}$ em que a pressão diminui para 7,936 kPa. Finalmente a pressão se recupera para 13,459 $\mathrm{kPa}$, que é igual á pressão inicial, após $100 \mathrm{~h}$ de estática.

Tabela 1: Dados do teste isócrono (Lee, 1984).

\begin{tabular}{lccc}
\hline \multicolumn{1}{c}{ Períodos } & $\begin{array}{c}\text { Tempo } t, \\
\mathrm{~h}\end{array}$ & $\begin{array}{c}\text { Vazão } q_{g}, \\
\mathrm{x} 10^{3} \mathrm{~m}^{3} / \mathrm{d}\end{array}$ & $\begin{array}{c}\text { Pressão } p_{w f} \text { ou } p_{w s,}, \\
\mathrm{kPa}\end{array}$ \\
\hline shut in inicial & 48 & 0 & 13,459 \\
$1^{\circ}$ drawdown & 12 & 73,482 & 12,142 \\
$1^{\circ}$ shut in & 15 & 0 & 13,459 \\
$2^{\circ}$ drawdown & 12 & 93,265 & 11,680 \\
$2^{\circ}$ shut in & 17 & 0 & 13,459 \\
$3^{\circ}$ drawdown & 12 & 141,311 & 10,411 \\
$3^{\circ}$ shut in & 18 & 0 & 13,459 \\
$4^{\circ}$ drawdown & 12 & 178,052 & 9,101 \\
Fluxo estendido (estabilizado) & 72 & 169,574 & 7,936 \\
shut in final & 100 & 0 & 13,459 \\
\hline
\end{tabular}

$\mathrm{m}^{3} / \mathrm{d}$ = vazão do gás medido em condições standard de pressão (1 atm) e temperatura $\left(15^{\circ} \mathrm{C}\right)$.

Na Figura 9 a representação gráfica do comportamento das vazões e pressões do teste ao longo do tempo é mostrada.

Para a determinação do AOF, que é o objetivo deste trabalho, foi utilizado o

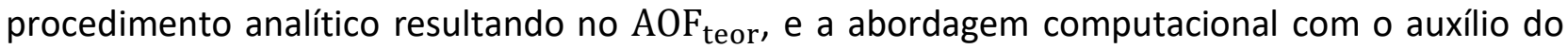
software IHS WellTest, que é identificado como $\mathrm{AOF}_{\mathrm{CAE}}$.(CAE - Computer Aided Engineering) $\mathrm{O}$ desempenho das duas abordagens foi comparado mediante o cálculo do erro definido mediante a seguinte equação

Erro $=\frac{\left|\mathrm{AOF}_{\text {teor }}-\mathrm{AOF}_{\mathrm{CAE}}\right|}{\mathrm{AOF}_{\text {teor }}}$. 
Os dados apresentados na Tabela 1 foram inseridos no software IHS WellTest. O uso da ferramenta foi possível por que o Laboratório de simulação numérica da Engenharia de Petróleo (Labsim) da Ufes tem a licença acadêmica cedida pela empresa IHS Markit.

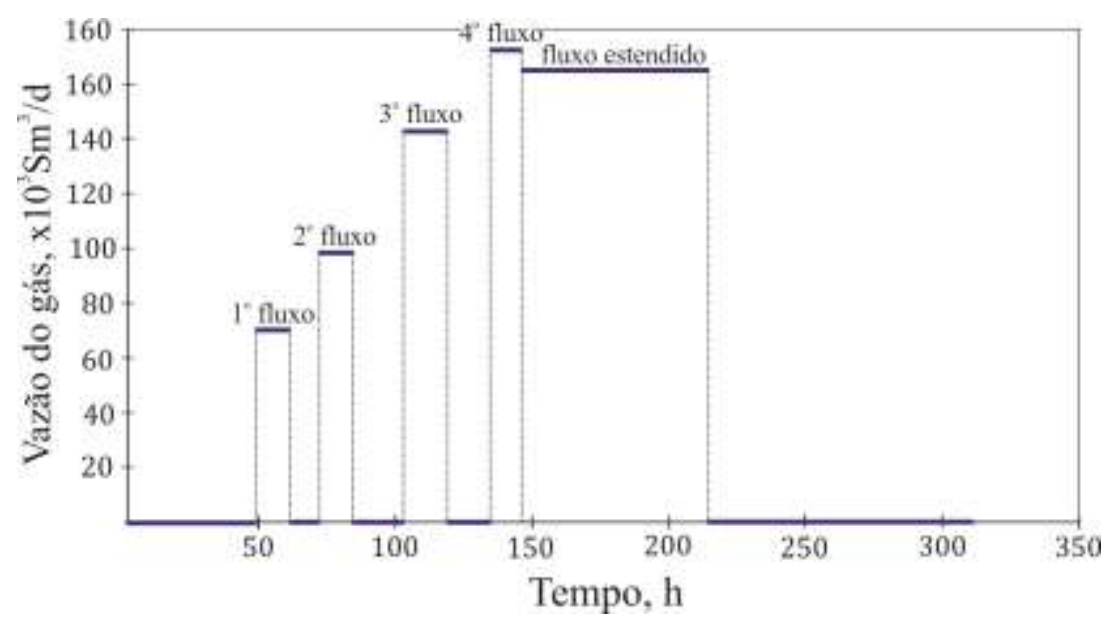

(a)

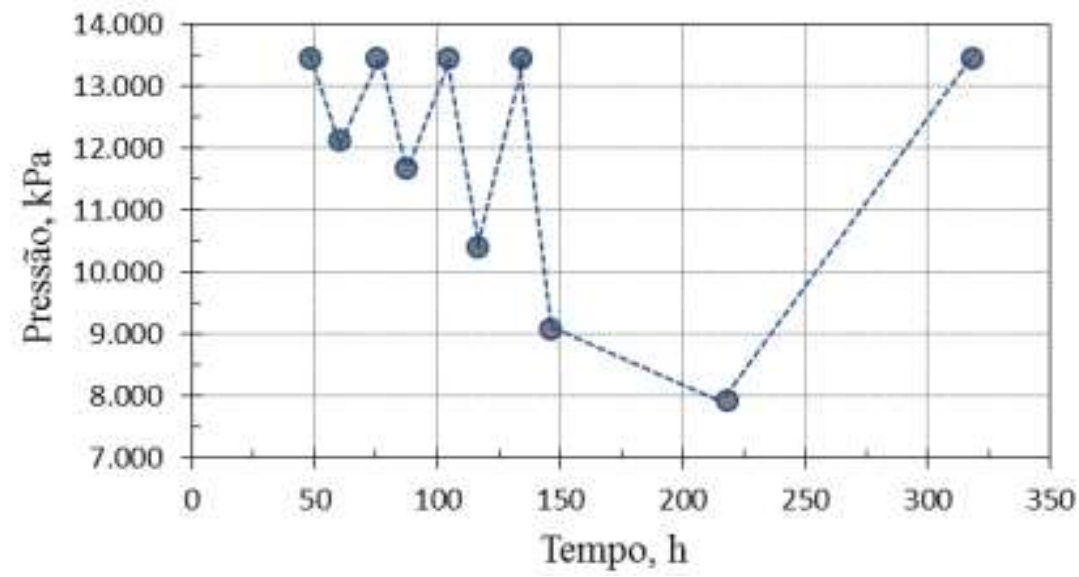

(b)

Figura 9: Representação gráfica dos dados do teste de poço: (a) variação da vazão com o tempo; (b) variação da pressão com o tempo.

\section{RESULTADOS E COMENTÁRIOS}

Nesta seção são apresentados os resultados obtidos utilizando como base os dados de períodos de fluxo e de estática de um teste isócrono para o escoamento de gás em um reservatório apresentados por de Lee (1984). São utilizados as abordagens analítica e computacional para determinar o AOF teórico ( $\mathrm{AOF}_{\text {teor}}$ ) e computacional ( $\mathrm{AOF} \mathrm{F}_{\mathrm{CAE}}$ ).

\subsection{Método analítico}

Os dados dos quatro períodos de fluxo $\left(1^{\mathrm{er}}, 2^{\mathrm{do}}, 3^{\mathrm{er}}\right.$ e $4^{\circ}$ drawdown) da Tabela 1 são processados e reapresentados na Tabela 2. 
Tabela 2: Processamento dos dados do período de fluxo do teste.

\begin{tabular}{ccc}
\hline $\begin{array}{c}\text { Vazão, } q_{g} \\
10^{3} \mathrm{Sm}^{3} / \mathrm{d}\end{array}$ & $\begin{array}{c}\bar{p}^{2}-p_{w f}^{2} \\
(\mathrm{kPa})^{2}\end{array}$ & $\begin{array}{c}\left(\bar{p}^{2}-p_{w f}^{2}\right) / q_{g} \\
10^{-3}(\mathrm{kPa})^{2} /\left(\mathrm{m}^{3} / \mathrm{d}\right)\end{array}$ \\
\hline 73,482 & 33,7129 & 0,4602 \\
93,265 & 44,7172 & 0,4810 \\
141,311 & 72,7423 & 0,5164 \\
178,052 & 98,3033 & 0,5538 \\
\hline
\end{tabular}

Os pares de pontos das colunas 1 e 3 da Tabela 2 são utilizados para elaborar o gráfico da Figura 10.

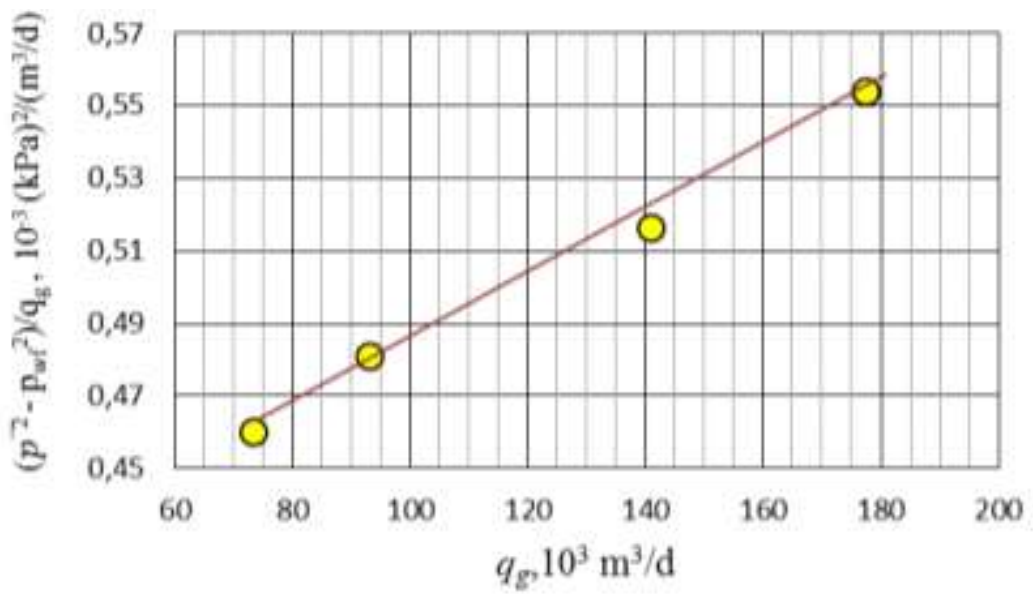

Figura 10: Dados do teste isócrono processados de acordo com a Equação (13) para determinar o AOF teor.

Com o gráfico da Figura 10 e utilizando os dois pontos que apresentem a maior queda de pressão, uma vez que representam o menor erro potencial (Lee, 1984), é determinado o coeficiente $b$ da Equação (13) a qual é reescrita por conveniência $\frac{\bar{p}^{2}-p_{w f}^{2}}{q_{g}}=a+b q_{g}$.

Os pontos $\left(q_{g} ; \frac{\bar{p}^{2}-p_{w f}^{2}}{q_{g}}\right)$ selecionados são $(141,311 ; 0,5164)$ e $(178,052 ; 0,5538)$, que permitem a montagem das duas equações seguintes

$0,5164 \times 10^{-3} \frac{(\mathrm{kPa})^{2}}{\left(\mathrm{~m}^{3} / \mathrm{d}\right)}=a+b\left(141,311 \times 10^{3} \frac{\mathrm{m}^{3}}{\mathrm{~d}}\right)$

$0,5538 \times 10^{-3} \frac{(\mathrm{kPa})^{2}}{\left(\mathrm{~m}^{3} / \mathrm{d}\right)}=a+b\left(178,052 \times 10^{3} \frac{\mathrm{m}^{3}}{\mathrm{~d}}\right)$,

resolvendo tem-se $b=0,001 \times 10^{-6}\left(\frac{\mathrm{kPa}}{\mathrm{m}^{3} / \mathrm{d}}\right)^{2}$.

Utilizando novamente a Equação (13), $\bar{p}^{2}-p_{w f}^{2}=a q_{g}+b q_{g}^{2}$, para os dados estabilizados $(s) q_{g s}=169,574 \times 10^{3} \mathrm{~m}^{3} / \mathrm{d}, p_{w f s}=7,936 \mathrm{kPa}, \bar{p}_{s}=13,459 \mathrm{kPa}$, também apresentados na Tabela 1, determina-se $a=\frac{\left(\bar{p}^{2}-p_{w f}^{2}\right)_{s}-b q_{g s}^{2}}{q_{g s}}=0,5273 \times 10^{-3} \frac{(\mathrm{kPa})^{2}}{\mathrm{~m}^{3} / \mathrm{d}}$.

Com $a$ e $b$ calculados a partir dos dados do teste, a Equação 13 assume a forma 


$$
\bar{p}^{2}-p_{w f}^{2}=\left[0,5273 \times 10^{-3} \frac{(\mathrm{kPa})^{2}}{\left(\mathrm{~m}^{3} / \mathrm{d}\right)}\right] q_{g}+\left[0,001 \times 10^{-6}\left(\frac{\mathrm{kPa}}{\mathrm{m}^{3} / \mathrm{d}}\right)^{2}\right] q_{g}^{2} .
$$

A última etapa é determinar o AOF, obtido na Equação (18) por definição para a pressão no fundo de poço igual a $p_{w f s}=0,0 \mathrm{kPa}$ e $q_{g}=\mathrm{AOF}$. Utilizando também a pressão média do reservatório $\bar{p}_{s}=13,459 \mathrm{kPa}$ e organizando obtém-se a Equação (19)

$0,001 \times 10^{-6}\left(\frac{\mathrm{kPa}}{\mathrm{m}^{3} / \mathrm{d}}\right)^{2} \mathrm{AOF}^{2}+0,5273 \times 10^{-3} \frac{(\mathrm{kPa})^{2}}{\mathrm{~m}^{3} / \mathrm{d}} \mathrm{AOF}-(13,459 \mathrm{kPa})^{2}=0$,

que é uma equação de segundo grau e cuja solução é obtida pela fórmula de Bhaskara, resultando em $\mathrm{AOF}=237,000 \times 10^{3} \mathrm{~m}^{3} / \mathrm{d}$ que é o $\mathrm{AOF}_{\text {teor }}$.

\subsection{Método computacional (CAE - Computer Aided Engineering)}

Os dados da Tabela 1 são utilizados para alimentar o software IHS WellTest. Após seguir diversos procedimentos próprias da ferramenta numérica, conforme comentado me Garuzzi e Romero (2014), gera-se a Figura 11 em escala log-log de $\Delta p^{2} v s . q_{g}$, onde $\Delta p^{2}=\bar{p}^{2}-p_{w f}^{2}$.

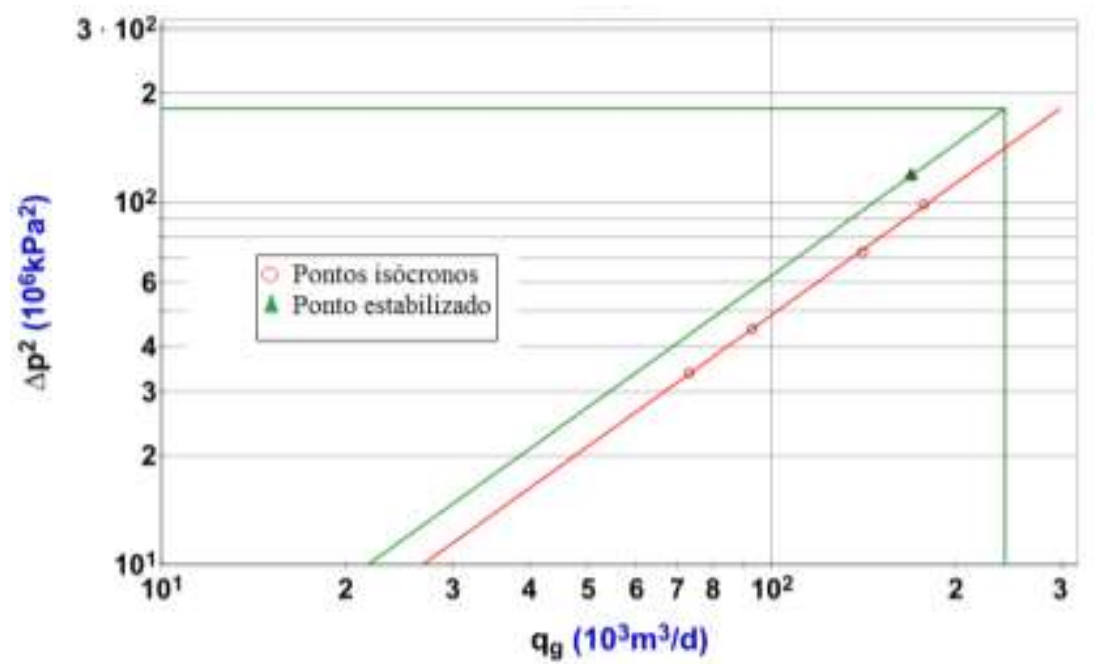

Figura 11: Gráfico log-log de $\Delta \mathrm{p}^{2}$ vs. qg.

A partir do gráfico da Figura 11 são obtidos diretamente os valores de $n, C$ e $A_{\text {OOF, }}$ os quais são apresentados na Tabela 3.

Tabela 3: Valores dos parâmetros obtidos pelo gráfico apresentado na Figura 11.

\begin{tabular}{cc}
\hline Parâmetro & Valor obtido \\
\hline$n$ & 0,830 \\
$C,\left(10^{3} \mathrm{~m}^{3} / \mathrm{d}\right) / 10^{6}\left(\mathrm{kPa}^{2}\right)^{\mathrm{n}}$ & 33,97 \\
$\mathrm{AOF}_{\mathrm{CAE}}\left(10^{3} \mathrm{~m}^{3} / \mathrm{d}\right)$ & 240,992 \\
\hline
\end{tabular}

O software também permite gerar a curva de IPR a partir da pressão média do reservatório, $\bar{p}$. Conforme discutido, a curva IPR é uma representação gráfica do comportamento da pressão medida em frente aos canhoneados para diferentes vazões do gás, no reservatório. A Tabela 4 mostra esta relação sendo a Figura 12 a curva IPR propriamente dita. 
Tabela 4: Dados para gerar a curva IPR.

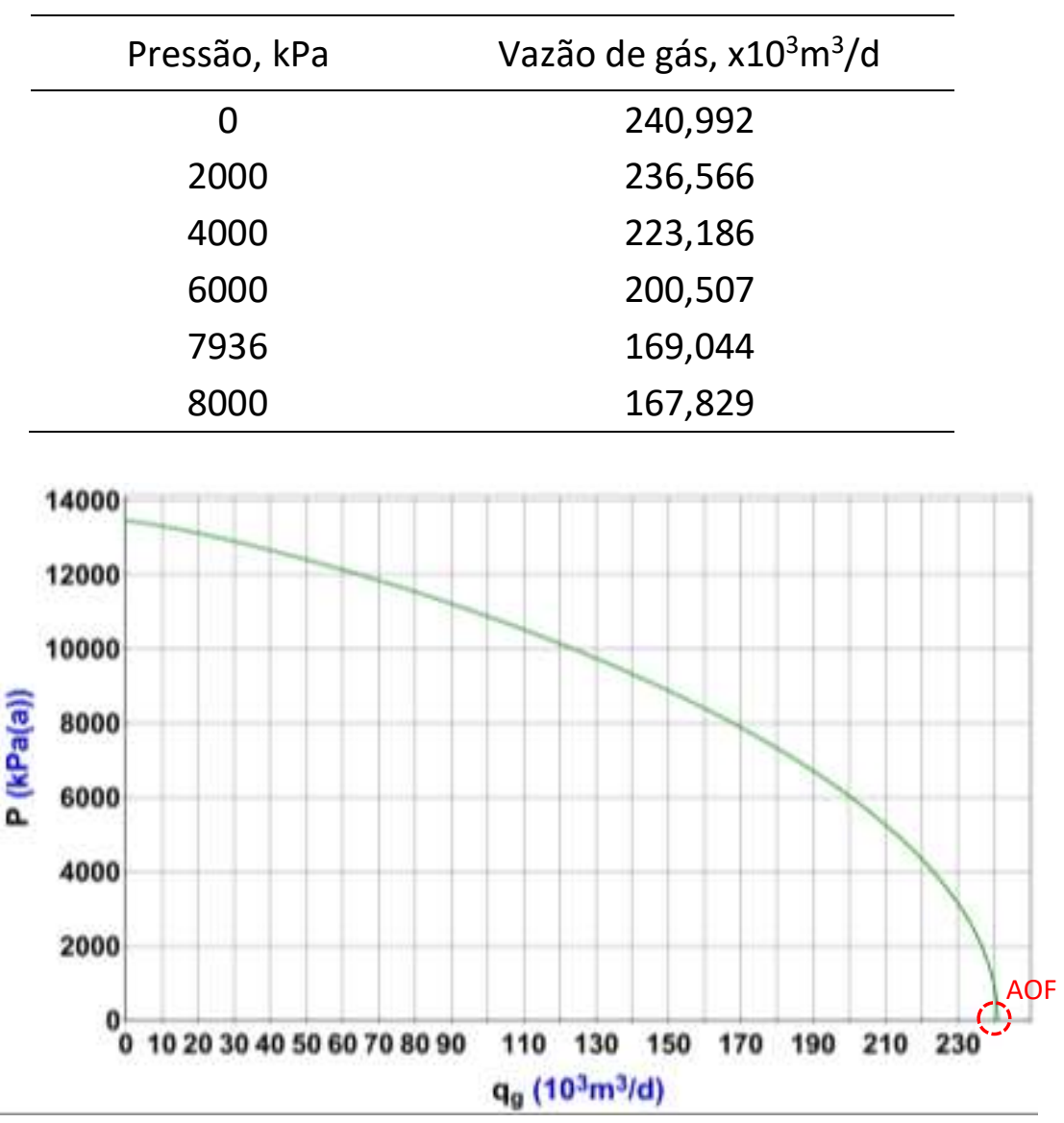

Figura 12: Gráfico da curva IPR obtida com os dados da Tabela 2.

A curva IPR mostra toda sua utilidade quando apresentada juntamente com a curva TPR (Tubing Performance Relationship). A TPR é a relação da pressão e vazão de fluidos no interior da coluna de produção. Isso porque do lado do reservatório objetivando uma maior vazão de gás, a pressão no fundo $\left(p_{w f}\right)$ deve ser a menor possível (Figura 12). No lado da coluna de produção, a vazão de gás é maximizada se a mesma pressão no fundo $\left(p_{w f}\right)$ for a menor possível. Existe portanto uma oposição de solicitações. O equilíbrio é encontrado no ponto de intersecção das duas curvas quando apresentadas no mesmo gráfico.

\subsection{Comparação}

Para cada abordagem foi encontrada um valor de AOF (Absolute Open Flow) diferente, $\mathrm{AOF}_{\text {teor }}=237,000 \times 10^{3} \mathrm{~m}^{3} / \mathrm{d}$ e $\mathrm{AOF}_{\mathrm{CAE}}=240,992 \times 10^{3} \mathrm{~m}^{3} / \mathrm{d}$; porém o erro associado calculado a partir da Equação (15), é de 1,7\%. Esse erro se deve ao fato de que no método computacional, o AOF é estimado através de uma aproximação gráfica que é função direta do cuidado no uso do software assim como da experiência do usuário com o aplicativo.

A aplicação da abordagem analítica requer conhecimentos teóricos específicos que não são simples de serem adquiridos e dominados. O uso do simulador requer ter acesso a ele. Softwares acadêmicos, gratuitos, que efetuem estas tarefas não se encontram disponíveis. 0 segundo desafio no simulador é compreender seu funcionamento. Vencidas estas etapas o software retorna a resposta muito mais rápido, mas em certa forma perde-se contato com a essência da modelagem matemática própria da abordagem analítica. 
No caso relativamente simples, pela reduzida quantidade de dados, abordado neste artigo, não se destaca a vantagem e poder do simulador comercial. Quando se trata de situações reais em que dados são aquisitados em taxas de segundos, ou minutos, ou horas e ao longo de semanas, o cenário muda drasticamente. O processamento manual torna-se inviável e o simulador passa a ser de uso padrão.

A finalidade deste estudo é compreender a modelagem matemática implementada nos simuladores comerciais. A comparação dos resultados mostra que houve sucesso na proposta.

\section{CONCLUSÕES}

Embora os testes de poços sejam muito importantes para iniciar ou dar continuidade ao desenvolvimento de um campo, existe uma carência de informações sobre o tema no idioma português (Romero \& Garuzzi, 2015). Esta escassez pode ser explicado porque testes de poços lidam com informações confidenciais de companhias operadoras: existência ou não de hidrocarbonetos, e se eles existem quais são seus volumes. Informações desta ordem estão associadas com o valor comercial das empresas petrolíferas e há extremo interesse em serem preservadas.

A aquisição de dados é um desafio importante pois é realizado em campo lidando com situações incomuns e muitas perigosas para os operadores, para a imagem da empresa e para o meio ambiente. $O$ processamento e interpretação dos dados, que é a etapa posterior à coleta de informação, exige conhecimentos da teoria de meios porosos e outras áreas da engenharia de petróleo, onde são utilizadas técnicas como a analítica, a computacional com auxílio de um software especializado, e muitas vezes ferramentas como as curvas tipo (Bourdet, 2002).

Este trabalho traz uma discussão sobre os fundamentos dos testes de pressão com direcionamento para o teste isócrono, que é específico para reservatórios de gás. Na situação analisada, os métodos analítico e computacional considerados, apresentam uma diferença menor do que $2 \%$ entre eles na determinação do AOF. Um melhor ajuste da curva que contém os pontos transientes no software IHS WellTest pode diminuir e diferença entre os valores encontrados. Isso depende da experiência do usuário (Garuzzi \& Romero, 2014).

Cada método tem pontos favoráveis e desfavoráveis. A abordagem analítica dispensa o uso de software comercial e é por tanto de execução rápida, precisa e traz economia de recursos. Entretanto, tarefas que são simples com o uso do software, tornam-se complicadas e cansativas quando feitas na mão. A ferramenta CAE - Computer Aided Engineering (Engenharia Auxiliada por Computador), apresenta uma gama enorme de possibilidades, entretanto sua curva de aprendizado é lenta. São, como mínimo, 12 meses de uso continuo para conhecer e saber explorar as diversas possibilidades oferecidas. Desta forma, quando existe domínio da ferramenta e acesso a ela, a resolução do problema pelo método computacional é preferível, já que demanda menos tempo. Entretanto, é importante destacar que a física do processo é melhor entendida quando as operações são realizadas do modo tradicional, utilizando caneta e papel. 


\section{AGRADECIMENTOS}

Os autores agradecem à IHS Markit pelo fornecimento das licenças acadêmicas do software comercial IHS WellTest. Nosso agradecimento também ao capítulo estudantil SPE Ufes <spe.ufes.br> pela obtenção do acesso gratuito à plataforma OnePetro da SPE - Society of Petroleum Engineers e ao Labsim - Laboratório de Simulação Numérica da Engenharia de Petróleo, instalações utilizadas para efetuar o presente trabalho

\section{REFERÊNCIAS BIBLIOGRÁFICAS}

Agarwal, R.G., Al-Hussainy, R., \& Ramey H.J. Jr. (1970). An investigation of wellbore storage and skin effect in unsteady liquid flow: I. Analytical treatment (SPE-2466-PA). Society of Petroleum Engineers. SPE-2466. DOI: 10.2118/2466-PA.

Ahmed, T., \& Meehan, D.N. (2012). Advanced Reservoir Management and Engineering. 2. Ed. Massachussets: Editora Elsevier.

Al-Hussainy, R., \& Ramey, H.J. Jr. (1966). Application of real gas flow: theory to well testing and deliverability forecasting. Journal of Petroleum Technology.

Bourdet, D. (2002). Well test analysis: the use of advanced interpretation methods. 1. ed. Amsterdam. Elsevier.

Bourdet, D.P., Whittle, T.M., Douglas, A.A., \& Pirard, Y.M. (1983). A new set of type curves simplifies well test analysis. World Oil 196.

Fetkovich, M.J. (1973). The isochronal testing of oil wells. Paper SPE 4529, Presented at the SPE Annual Meeting, Las Vegas, Nevada, Sept. 30-Oct. 3.

Garuzzi, R.P., \& Romero, O.J. (2014). Abordagem analítica e computacional do teste drawdown. Latin American Journal of Energy Research, v. 1, p. 39-45. DOI: 10.21712/lajer.2014.v1.n1.p39-45.

Gringarten, A.C., Bourdet, D.P., Landel, P.A., \& Kniazeffi, V.J.A. (1979). Comparison between different skin and wellbore storage type-curves for early-time transient analysis. Society of Petroleum Engineers, SPE- 8205.

Guiteras, O.H. (2003). Metodologia de análise global para o desenvolvimento de um campo de gás natural. Dissertação de Mestrado. Engenharia mecânica. PUC-RIO.

HIS WellTestTM Help Guide. (2015). Fekete Associates, Inc@.

Lee, J. (1984). Well Testing. SPE Textbook Series, v. 1.

Matthews, C.S., Brons F., \& Hazebroek, P.A. (1954). Method for determination of average pressure in a bounded reservoir. American Institute of Mining, Metallurgical, and Petroleum Engineers. AIME 201. 
Miller, C.C., Dyes, A.B., \& Hutchinson, C.A. (1950). The estimation of permeability and reservoir pressure from bottom hole pressure build-up characteristics. American Institute of Mining, Metallurgical, and Petroleum Engineers. AIME 189.

Romero, O.J. (2015). Notas de aula da disciplina Avaliação de Formações ministrada em 2015/2. Graduação em Engenharia de Petróleo, Ufes.

Romero, O.J., \& Garuzzi R.P. (2015). Testes de pressão em reservatórios de óleo e gás (ISBN: 978613-0-15846-0). Novas edições acadêmicas, v. 1, 113p.

Rosa, A.J., Carvalho, R.S., \& Xavier, J.A.D. (2006). Engenharia de reservatório de petróleo. 2ª ed. Rio de Janeiro: Interciência, 832p. 